\title{
THE STILES-CRAWFORD COLOUR CHANGE
}

\author{
W. WiJngaARd, M. A. Bolman and F. Budding \\ Department of Medical and Physiological Physics. State University Utrecht. Ltrecht. the Netheriands
}

(Received 18 July 1973)

\begin{abstract}
To obtain the Stiles-Crawford colour change. the absorption of guided and unguided light by a cone outer segment as a function of the angle of incidence is estimated. The model is specified in two ways: the $H E_{1.1}$ model. based on a waveguide modal analysis. and the geometrical optics model. The measurements by Stiles (1937) are fitted with reasonable success. both for the intensity effect and for the colour change. using the phenomenon of selfscreening to explain the colour change.
\end{abstract}

The Stiles-Crawford colour change has been explained in the literature as due to selfscreening of the visual pigment (Brindley, 1953; Walraven and Bouman, 1960). Walraven and Bouman (1960) have obtained a specific expression for the absorption of light by the photopigment. attributing the Stiles-Crawford intensity effect to leakage from the outer segment and leakage from the inner segment. In this paper the Stiles-Crawford intensity effect is attributed to leakage from the inner segment. The expression for the absorption of light by the pigment in the outer segment. which will be used here. is specified in two ways. In the geometrical optics model the parameters. except the absorption coefficients. are not wavelength-dependent. In the $H E_{1.1}$ model the parameters are obtained from the characteristics of the $H E_{1,1}$ dielectric waveguide mode. The Stiles-Crawford colour change is attributed to selfscreening.

\section{THE GENERAL EXPRESSION}

From ray tracing and microwave model experiments it is probable that the Stiles-Crawford intensity effect is caused by the leakage of light from the inner segment. The light leaking from an inner segment may strike an outer segment. When the light is incident along the axis of a cone a large part will be guided through the whole length of the outer segment. but when the light is directed obliquely to the retina the unguided light becomes important. Thus, for incidence along the axis of the cone the light passes a higher density of pigment than for oblique incidence, which results in a colour change due to the phenomenon of solfscreening.

To obtain quantitative results some conditions have to be specified. It will be assumed that the cones are directed to the centre of the exit pupil and that each cone occupies one unit cell, with area $S_{1}$, of a hexagonal lattice.

Let $P_{t}$ be the flux incident on each unit cell. with angle $\theta_{i}$, between the propagation vector $\mathbf{k}_{i}$ and the axis of a cone (Fig. 1). $P\left(\theta_{0}\right)$ is defined to be the fraction of $P$, that is guided by the outer segment. It is assumed that the part $k\left(\theta_{13}\right)$ of the guided light is guided inside the outer segment, to account for the fact that the flux in a dielectric waveguide mode is only partly inside the guide.

When $x$ is the absorption coefficient of the pigment. the absorption in the outer segment due to the guided light is

$$
P\left(\theta_{0}\right) P_{l}\left(1-\exp \left(-\kappa\left(\theta_{0}\right) \times l\right)\right)
$$

where $l$ is the length of the outer segment (Snyder, 1972). It will be assumed that the unguided light is propagating in the direction $\mathbf{k}_{\boldsymbol{s}}$ (Fig. 1) with an angle $\theta$ between the propagation direction and the axis of a cone. and that this light is homogeneously distributed in the outer segment layer. The absorption due to the unguided light is calculated using geometrical optics. neglecting reflection and refraction of the rays in the outer segment layer. and assuming that the absorption coefficient and the maximum length $d$ of the pigment crossed by the rays are small enough that exp $(-x d)$ may be approximated by $1-\alpha d$. When a ray incident on an outer segment has travelled through another outer segment, the reduction of the intensity of the ray is neglected. With these assumptions, the light absorbed by the outer segment from the unguided field is given by $I_{11} \times l^{\prime}$. Here $l$ ' is the volume of the outer segment and $I_{I}$ is the flux density of the unguided light.

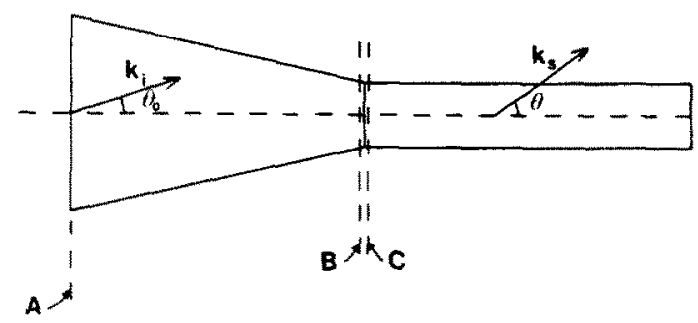

Fig. 1. Schematic drawing of a cone. 
For $x=0$ the flux incident on the lattice and the flux transmitted by the lattice are equal and therefore

$$
P_{1}=P\left(\theta_{0}\right) P_{1}+I_{u} \cos (\theta) S_{1}
$$

here $S_{1}$ is the area of the unit cell of the hexagonal lattice.

So $\left.I_{n}=P_{1} ; 1-P\left(\theta_{i}\right)\right)_{i} /\left(\cos (\theta) S_{1}\right)$, and the absorption due to the unguided light is given by

$$
\frac{\left(1-P\left(\theta_{0}\right)\right) P_{1}}{\cos (\theta)} \cdot \frac{\pi p^{2}}{S_{1}} x 1
$$

where $\rho$ is the radius of the outer segment. Therefore the total absorption is given by

$$
P_{I}\left\{P\left(\theta_{0}\right)\left(1-\exp \left(-\kappa\left(\theta_{0}\right) \alpha l\right)\right)+\frac{1-P\left(\theta_{0}\right)}{\cos (\theta)} \cdot \frac{\pi p^{2}}{S_{1}} x l_{1} .\right.
$$

where $P_{1}=I_{1}, S_{1} \cos \left(0_{0}\right)$. and $I_{0}$ is the flux density in the incident field. For relatively large angles of incidence $P\left(\theta_{0}\right)$ is small compared with 1 and the absorption is approximately given by

$$
I_{0} S_{1} \frac{\cos \left(\theta_{0}\right)}{\cos (\theta)} \cdot \frac{\pi \rho^{2}}{S_{1}} x l .
$$

It should be emphasized that in general the absorption is nonzero in this limit.

The parameters to be specified are $P\left(\theta_{0}\right), \kappa\left(\theta_{0}\right)$. xl. $\theta$. and $\pi f^{2}: S_{1}$. The choice of $P\left(\theta_{0}\right)$ and of $k\left(\theta_{0}\right)$ will be discussed with the specific models. The value of $x l$ is given by its maximum value $(x)_{\max }$ the wavelength $\lambda_{\operatorname{mix}}$ for which this maximum is obtained and

$$
x_{r a l}=\frac{(x l)}{(x l)_{\max }} \text { as a function of }\left(\frac{1}{\lambda}-\frac{1}{\lambda_{\max }}\right) \text {. }
$$

For the blue cone system $x_{\text {rel }}$ is obtained from the rhodopsin absorption curve given by Wyszecki and Stiles (1967). For the red and green system $\dot{x}_{\mathrm{rel}}$ is obtained from the $G$ sensitivity function at the retinal level given by Vos (1972) by transforming it to an equal quantum spectrum. The $G$ function was thus used as a pigment absorption function (Smith and Pokorny, 1972). The absorption by the lens and macular pigment was included in the calculations. The numerical values were taken from W yszecki and Stiles (1967). A correction for the different pathlengths through the lens for different excentricities was applied following Weale (1961) (see also Mellerio. 1971). In the following discussion for the red, green and blue cone systems $\lambda_{\max }$ was chosen to be 562.0 .535 .5 and $435.0 \mathrm{~nm}$ respectively. The colour change was calculated in terms of the red and green chromaticity coordinates of a W.D.W. system with primaries 6452.526 .3 and $444.4 \mathrm{~nm}$, and normalizing wavelengths 579.7 and $487.8 \mathrm{~nm}$ (Enoch and Stiles, 1961). For convenience the colour change for monochromatic light of wavelength $\lambda_{0}$ was expressed in terms of an apparent wavelength shift $\Delta \lambda$. To this end the point of intersection was calculated between the spectrum locus for perpendicular incidence and the straight line joining the equi-energy white point (wavelength basis) and the point representing the oblique incident light. When the point of intersection is given by $i_{i t}, \Delta i$ is defined by $\Delta i=i_{H_{1},}-i_{i l}$, Enoch and Stiles. 19611 .

The parameter $(x)_{\text {max }}$ was given the same value for the three cone systems. In all calculations $|\theta|=$ $\left|\theta_{3}\right|+0.1$ (radians). To obtain the luminance, the responses of the systems were added up with a weight for the red. green and biue system equal to 0.91, 0.54 and 0.055 . respectively. With these parameters an approximate fit with the photopic relative luminous efficiency function $k_{i}$ is obtained.

\section{THE GEOMETRICAL OPTICS MODEL}

In this model. which assumes the specific effects of the presence of waveguide modes to be small. $k\left(\theta_{0}\right)$ is assumed to be equal to 1 . A good choice for the function $P\left(\theta_{0}\right)$ is $P\left(\theta_{0}\right)=P_{0} \exp \left(-\beta \theta_{0}^{2}\right)$. The parameter $\beta$ gives the directionality of the inner segment. No attempt was made to calculate $P\left(\theta_{0}\right)$ from a geometrical optics model of the cone inner segment. It was possible to obtain an approximate fit with the results for the intensity and colour effect by Stiles (1937). To fit Stiles' results, $P_{1}$ was chosen to be 0.8 and $(\alpha i)_{\max }=$ 1.3 for the three cone systems. $\pi \rho^{2} / S_{1}$ was chosen to be 0.083 for the red and green system. It was necessary to choose $\pi \rho^{2} / S_{1}<0.083$ for the blue cone system to be able to obtain a positive $\Delta \hat{i}$ in the blue part of the spectrum. A value of $\pi \rho^{2} / S_{1}=0.052$ was chosen.

When $\beta$ was equal to 110 for the three systems, an approximate fit with the Stiles-Crawford intensity effect was obtained. However in this case the $\Delta \lambda$ curves for 579 and $457 \mathrm{~nm}$, for example are too broad compared with the experimental curves; and the observed oscillation in the $\Delta i$ curve for $522 \mathrm{~nm}$ was not obtained. The ultimate choice for the parameter $\beta$ was 103.110.130 for the red, green and blue system respectively. A summary of the parameters is given in Table 1.

In Fig. 2 the dots indicate the experimental results for the parameter $h(\lambda)$ as determined by Stiles (1937) and given in his Table II: the continuous curve was calculated using the geometrical optics model with the parameters of Table 1 . The experimental results by Stiles were obtained from the sensitivity $s$ for a point $275 \mathrm{~mm}$ from the point of maximum sensitivity in the pupil and from the sensitivity $s_{0}$ for the point of maximum sensitivity, assuming for $s$ a relation of the form

Table 1. Parameters for the geometrical optics model fitting approximately the results of Stiles (1937)

\begin{tabular}{lcccc}
\hline & $(x)_{\operatorname{mix}}$ & $\pi)^{2} / S_{1}$ & $f$ & $P_{0}$ \\
\hline Red & $1 \cdot 3$ & 0.083 & 10.3 & 0.8 \\
Green & 13 & 0.083 & 110 & 08 \\
Blue & $1 \cdot 3$ & 0.052 & 100 & 0.8 \\
\hline
\end{tabular}




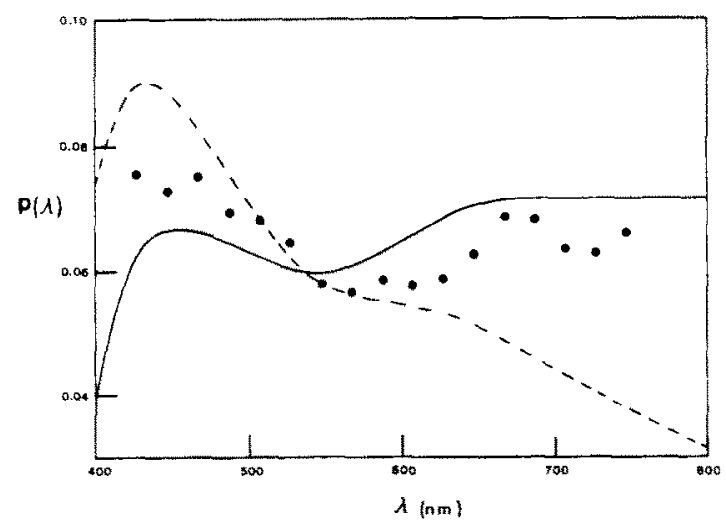

Fig. 2. Results for $p(\lambda)$.

- Experimental values of Stiles (1937)(from his Table II).

Results obtained with the geometrical optics model with the parameters of Table 1 .

- - Results obtained with the $H E_{1,1}$ model with the parameters of Table 3 .

$s=s_{f} 10^{-}$mhere $r$ is the distance in millimeters from the point of maximum sensitivity. From the model $p(\lambda)$ is obtained in the same way, assuming $\theta_{0}=$ $0045, \mathrm{rrad}$.

In Fig. 3 the sensitivity $\eta$ is given for $\lambda_{i}=440 \mathrm{~nm}$ and for $\lambda_{n}=540 \mathrm{~nm}$ as a function of the point of incidence of the light along a straight line in the entrance pupil. The coordinate along that line is $d$. The experimental points by Stiles (1937) from his Table I are given by the circles: these results were obtained for a traverse through the centre of the pupil along a line $0.5 \mathrm{~mm}$ from the point of maximum sensitivity. For the centre of the pupil $d=-0.5 \mathrm{~mm}$.

The continuous curves give the results for the geometrical optics model with the parameters given in

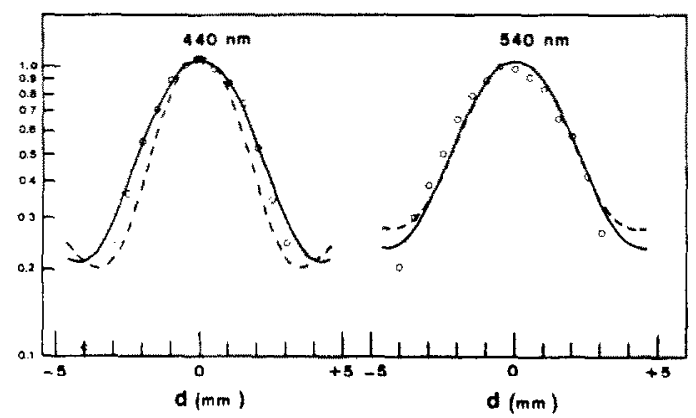

Fig. 3. The sensitivity $\eta$ along a traverse through the pupil for $\lambda=440 \mathrm{~nm}$ and $\lambda=540 \mathrm{~nm}$.

0000 Experimental results of Stiles (1937) for a horizontal traverse through the centre of the pupil. For the centre of the pupil $d=-0.5 \mathrm{~mm}$.

Calculated from the geometrical optics model with the parameters of Table 1 for a pupil traverse $0.5 \mathrm{~mm}$ from the point of maximum sensitivity in the pupil.

Calculated in the same way using the $H E_{1,1}$ model with the parameters of Table 3 .

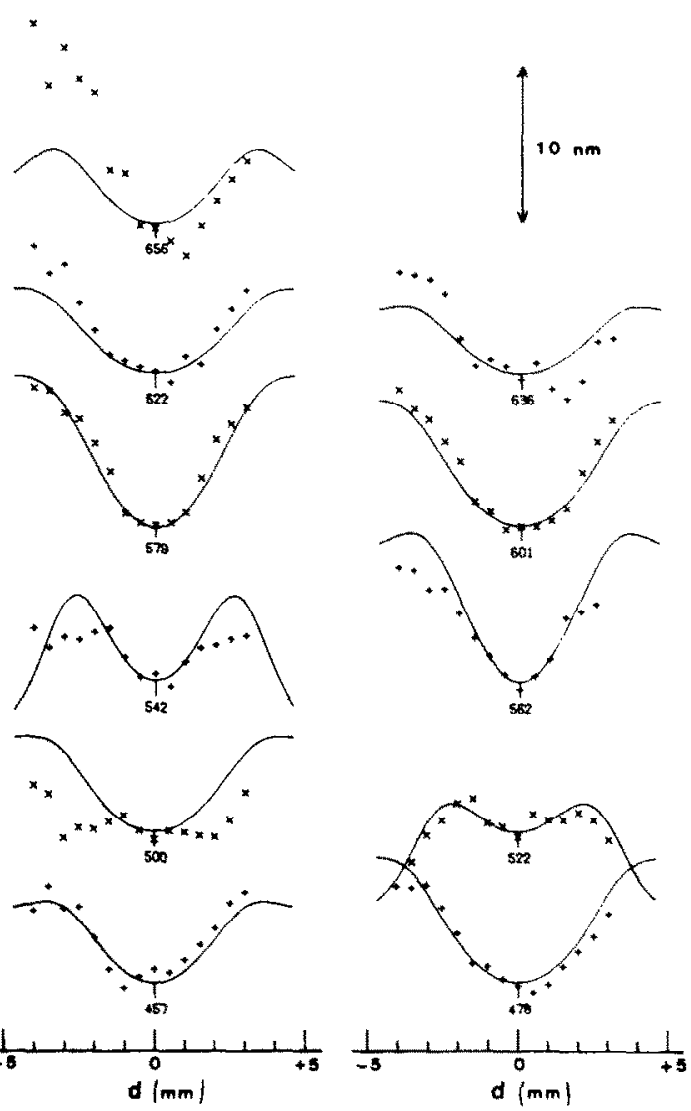

Fig. 4. Colour change in terms of the wavelength shift $\Delta \lambda$. $x$ and + experimental results of Stiles (1937) from his Table IV for a traverse through the centre of the pupil. For the centre of the pupil $d=-0.5 \mathrm{~mm}$.

Results from the geometrical optics model with the parameters of Table 1 for a traverse $0.5 \mathrm{~mm}$ from the point of maximum sensitivity in the pupil.

Table 1; these results are for a pupil traverse $0.5 \mathrm{~mm}$ from the point of maximum sensitivity in the pupil. The maximum sensitivity along the traverse was assumed to be obtained for $d=0$. In both cases $\eta$ was normalized to 1 for $d=-0.5 \mathrm{~mm}$.

In Fig. 4 the colour change is given in terms of the wavelength shift $\Delta \lambda$ as a function of the coordinate $d$ along the pupil traverse. The experimental results are from Tabie IV by Stiles (1937), again assuming for the centre of the pupil $d=-0.5 \mathrm{~mm}$. The continuous curves are calculated for a traverse $0.5 \mathrm{~mm}$ from the point of maximum sensitivity in the pupil, using the geometrical optics model with the parameters given in Table 1. In the experiments by Stiles the wavelength of the variable beam was adjusted to give the same hue as a beam entering the eye through the centre of the pupil $(d=-0.5 \mathrm{~mm})$. Therefore the calculated curves were displaced in the vertical direction so that $\Delta \lambda=0$ for $d=-0.5 \mathrm{~mm}$. 


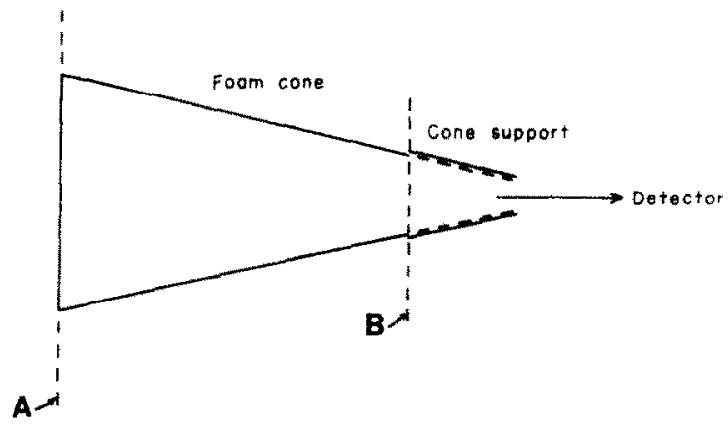

Fig. 5. Schematic drawing of the model of Enoch and Fry (1958) and Enoch (1960).

The value $(x \mid)_{\max }=1.3 \mathrm{implies}$ an O.D. for the cones of 0.565 , in accordance with some estimates of pigment density (Miller, 1972; King-Smith. 1973a, 1973b).

\section{THE $H E_{1,2}$ MODEL}

This model was inspired by the microwave model experiments by O'Brien (1951). Enoch and Fry (1958). and Enoch (1960). First we will analyze these experiments. The model by Enoch and Fry (1958) and by Enoch (1960) consisted of a polystyrene foam cone mounted on a metal horn (Fig. S). To characterize the electromagnetic properties of the model the cutoff parameter $t$ of the cylindrical dielectric waveguide will be used here. Here $r=(2 \pi \rho / \lambda)\left(n_{1}^{2}-n_{2}^{2}\right)^{12}$ with $\rho$ the radius of the guide, $;$ the vacuum wavelength of the wave and $n_{1}$ and $n_{2}$ the refractive index of the core and the matrix medium respectively. The cone is characterized by two values of $t, v_{A}$ the value of $v$ at plane $A$ and $c_{B}$ the value of $v$ at plane $B$ (Fig. 5). For the model by Enoch $(1960)$, when $i=2.36 \mathrm{~cm}, v_{A}=3.8$ and $v_{B}=$ 1.27. In this case the guided modes possible at plane $A$ are the modes $H E_{1,1}, H E_{2,1}, T E_{0,1}$ and $T M_{0,1}$; at plane $B$ only the $H E_{1.1}$ mode is possible.

It was assumed that the coupling between these modes is negligible. so in this case the exact form of the conical portion of the inner segment has no influence on the results (Snyder. 1970).

A field incident on plane $A$ will excite the guided modes and also a radiation field. The $H E_{2.1}, T E_{0,1}$ and $T M_{t, 1}$ modes cannot be guided to plane $B$ because they have a cutoff value $v=2 \cdot 405$, and therefore the power in these modes will be coupled to the radiation field. The $H E_{1,1}$ mode however, may be guided to plane $B$. Therefore the field incident on the metal horn consists of the $H E_{1.1}$ mode and a part of the radiation field. For some of the experiments of Enoch (1960) the incident field was approximately a plane wave in the vicinity of the cone. In this case the power $I_{\mathrm{t}, \mathrm{f}}$ in the $H E_{1,1}$ mode may approximately be calculated using the method given by Snyder (1969). In the left part of Fig. 6 the continuous curve gives $l_{1.1}$ as a function of (1) $)_{r}^{2}$ for $r=3.8$. with unity incident power on the large end of the cone. Here $\theta_{r}=\theta_{1}$, $\delta$ with $\delta=1-n_{2}^{2} n_{1}^{2}$.
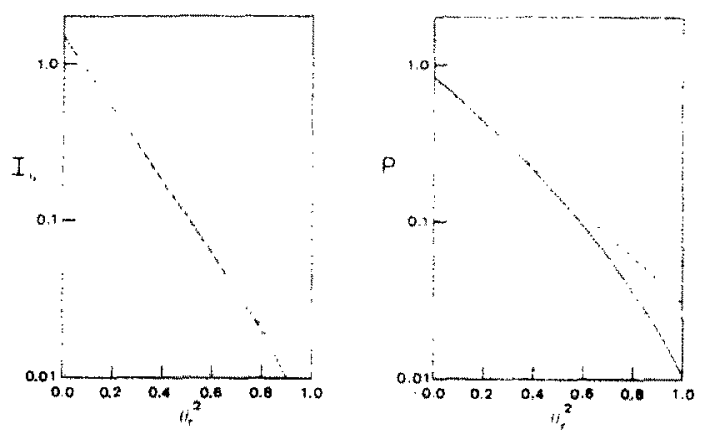

Fig. 6. Left part of the figure: results for the foam cone. The power $I_{t, 1}$ in the $H E_{1,1}$ mode excited by a plane wave with unity incident power on the large end of the cone. as a function of $\theta_{r}^{2}=\theta_{\delta}^{2} / \delta . r_{4}=3.8$.

$\therefore-B \cdot \exp \left(-\beta \theta_{0}^{2}\right)$.

Right part of the figure: results for the $H E_{1,1}$ model.

The fraction $P\left(\theta_{0}\right)$ of the light incident on a unit cell of the lattice that is guided to the outer segment. as a function of $\theta_{r}^{2}=\theta_{0}^{2} / \delta \cdot v_{i}=3 \cdot 8$.

$\cdots-P_{n} \exp \left(-\beta \theta_{0}^{2}\right)$.

In the same figure a function of the form $B \cdot \exp \left(-\beta \theta_{0}^{2}\right)$ is given by the interrupted curve showing the resemblance of both curves. $\beta$ and $B$ were calculated assuming the equality of both functions for $\theta_{0}=0$ and for $\theta_{0}=(u / v)_{k} \delta$. Here $u=\rho\left(k_{1}^{2}-h^{2}\right)^{12}, k_{1}$ is the propagation constant of plane waves in a medium of refractive index $n_{1}$ (the inner medium) and $h$ is the propagation constant of the $H E_{1,1}$ mode. The $H E_{1,1}$ mode is guided to plane $B$ by which the flux density becomes larger. For small angles of incidence the field on plane $B$ is therefore largely due to the $H E_{\mathrm{i}, 1}$ mode. This means that the left part of Fig. 6 gives approximately the power that is detected, in relative units, as a function of the angle of incidence of the plane wave for small angles of incidence. Enoch has approximated the experimental results for small $\theta_{13}$ with $s=$

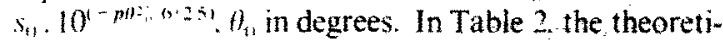
cal results are compared with Enoch's results, estimated from his Fig. 5.

The assumption that the $H E_{1,1}$ mode does not couple to the radiation field is not applicable when $v_{B}<1$. In this case the power inside the guide is only a small part of the total power in the $H E_{1,1}$ mode. The part of the experimental results of Enoch with $\lambda \geqslant 3.05 \mathrm{~cm}$ falls into this class and cannot be compared with theoretical results at this moment.

Table 2. The experimental values for $p(\lambda)$ obtained for the microwave model by Enoch (1960) compared with approximate theoretical results

\begin{tabular}{ccc}
\hline$\lambda(\mathrm{cm})$ & Enoch $(1960)$ & $\begin{array}{c}p \\
\text { theory }\end{array}$ \\
\hline 2.36 & 0.157 & 0.152 \\
2.63 & 0.111 & 0.137 \\
2.87 & 0.088 & 0.129
\end{tabular}


Table 3. Parameters for the $H E_{1,1}$ model fitting approximately the results of Stiles (1937)

\begin{tabular}{lccccc}
\hline & $(x \mid)_{\max }$ & $\pi \rho^{2} S_{1}$ & $r_{4}(700)$ & $r_{d}(700)$ & $j$ \\
\hline Red & 1.7 & 0.07 & 4.759 & 1.321 & 0.07 \\
Green & 1.7 & 0.07 & 4.929 & 1.368 & 0.07 \\
Blue & 1.7 & 0.042 & 5.471 & 1.176 & 0.07 \\
\hline
\end{tabular}

When the detector unit of the previously discussed microwave model is replaced by a dielectric rod with loss a model of a primate cone is obtained (Fig. 1). The photopigment in the outer segment will absorb a part of the flux of the guided field excited by the field incident on plane $C$ (Fig. 1) and a part of the flux of the radiation field. For the specific model discussed here it is assumed that the $H E_{1.1}$ mode is the only guided mode at the level of plane $B$. therefore $v_{B}<2.405$. It is assumed that in plane $A$ the cones are touching in at hexagonal lattice. therefore in plane $A$ the modes of the hexagonal array should be considered. As could have been anticipated $H E_{1,1}$ like array modes are indeed possible (Snyder and Kyhl, 1966). For the present purpose the excitation of the $H E_{1,1}$ mode by a plane wave incident on the retina was calculated using the method by Snyder (1969) for the $H E_{1.1}$ mode of the single guide with the plane wave truncated to a circle with an area equal to the area $S_{1}$ of the unit cell of the lattice. In this way the function $P\left(\theta_{0}\right)$ was obtained. $P\left(\theta_{0}\right)$ was approximated by the function $P_{0} \exp \left(-\beta 0_{0}\right)$ so that the functions are equal for $\theta_{0}=0$ and $\theta_{0}=$

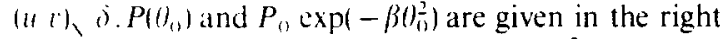
part of Fig. 6 for $r=3 \cdot 8$ as functions of $t_{r}^{2}$ with $\theta_{r}=$ $(1), i$.

It Wils assumed that the $H E_{1,1}$ mode incident on the outer segment will mainly excite the $H E_{1,1}$ mode of the outer segment. This assumption is justified when the difference between $r_{B}$ and $v_{C}$ is small. In this case $\kappa\left(\theta_{0}\right)$ is the part of the power of the $H E_{1.1}$ mode guided inside the outer segment. $k\left(\theta_{0}\right)$ is therefore independent of $t_{1}$, and in the following discussion will be indicated by $k$.

$k$ is the function indicated by $k_{n 1}$ in Fig. 11 of Snyder and Pask (1973). $P_{0}$ is approx 0.8 for the range of interest of $\imath_{A}$. For $l>2 \beta$ may be approximated by $\beta=0.22, \gamma$. Therefore $\beta$ is approximately given by

$$
\beta=0.22\left\{\frac{2 \pi \rho_{i} n_{1}}{i}\right\}^{2}
$$

where $\mu_{i}$ is the radius of the wide end of the inner segment.

The parameters of the $H E_{1,1}$ model for each cone system are $\left.\pi \rho^{2} ; S_{1}, \delta,(x)\right)_{\max }$, and the value $v_{A}(700)$ of $i_{i}$ and $r_{r}(700)$ of $r_{i}$ for the arbitrarily chosen wavelength of $700 \mathrm{~nm}$. These parameters are, however, not independent. For $S_{1}=1 \cdot 1 \pi \rho_{i}^{2}$; this implies that $v_{B}=$ $\left.i_{1}, 1 \cdot \mid \pi\right)^{2} S_{1} 1^{1}=$. The parameters have to be chosen in such a way that $i_{B}$ is less than 2.405 for the range of wavelengths of interest and that $v_{B}$ and $v_{C}$ do not differ much. $l_{c}$ determines $k$ and therefore the effective O.D. $k \cdot x /$ of the pigment in the cone outer segment.

To fit the experimental results by Stiles $11937 . I^{i c}$ was chosen equal to $t_{B}$. which implies that the refractive indices of the inner and outer segments are equal. $r_{c}(700)$ was chosen in such a way that $1<r_{c}<2.405$ for $800 \mathrm{~nm}>i>400 \mathrm{~nm}$. thus $1.142<i d(700)$ $<1.374$. The choice of the particular parameters in the $H E_{1,1}$ model was guided by the parameters for the geometrical optics model given in Table 1 . In particular the values for $\pi r^{2} S$, were only slightly changed. For the middle of the visible spectrum the values for $0.220 \%$ of the red and green system were chosen approximately equal to the respective values for $\beta$ of the geometrical optics model given in Table 1. The parameter $(x l)_{\text {mux }}$ was chosen in such a way that for the red and green system the maximum value of $k x /$ was approximately equal to $1 \cdot 3$, the value that had been used for the geometrical optics model. However. the value 0.8 for $P_{10}$ for the geometrical optics model was chosen in accordance with the $H E_{1,1}$ model. in which $P_{11}$ is approx 0.8 for the whok range of interest of $r_{4}$. The final set of parameters. used for the comparison with the experimental results of Stiles. is given in Table 3. The comparison was made in the same way as the comparison with the geometrical optics model.

The accuracy with which this model fits the experimental results for the colour effect is not much different from the fit obtained with the geometrical optics model. However, the function $p(\lambda)$ obtained with the $H E_{1,1}$ model is of a different kind. $p(\lambda)$ is given by the interrupted curve in Fig. 2. In Fig. 3, the sensitivity $\eta$ is given as a function of the point of incidence in the pupil by the interrupted curves for $\lambda=440 \mathrm{~nm}$ and $i=540 \mathrm{~nm}$.

From the parameters given in Table $3 \rho_{i}$ and $\rho$ may be obtained. assuming $n_{2}=1 \cdot 340$. The results are given in Table $4 . \rho_{i}$ is not equal for the three cone systems, the assumption that the cones are touching in a hexagonal lattice is therefore not completely justified. However. this assumption has only been used to calculate the area $S_{1}$. entering the calculations via the para-

Table 4. The radius $\rho_{i}$ of the wide end of the inner segment and the radius $\rho$ ol the outer segment obtained using the parameters for the $H E_{1,1}$ model from Table 3

\begin{tabular}{lll}
\hline & $\rho_{i}(\mu \mathrm{m})$ & $\mu(\mu \mathrm{n})$ \\
\hline Red & 1.442 & 0.400 \\
Green & 1.494 & 0.415 \\
Blue & 1.658 & 0.356 \\
\hline
\end{tabular}


meter $\pi \rho^{2} ; S_{1}$ and via the calculation of the power with which the $H E_{1,1}$ mode is excited.

From $\delta$ the refractive index $n_{\text {, may }}$ be obtained. again assuming $n_{2}=1.340$. The result is $n_{1}=1.390 \mathrm{in}$ accordance with the measurements by Sidman (1957) on some vertebrate cones.

\section{DISCLSSION}

The cone models given above are simplified in several respects. For example, mutual differences between the cones of one cone system are neglected. Differences in geometrical form and optical constants are not improbable. The results by Makous (1968) indicate differences in orientation of the cones. However. from the results by Coble and Rushton (1971) it is improbable that these differences are large.

The inner segment parts of the cone models given above are more general than Fig. 1 would suggest. For the geometrical optics model it has been assumed that the fraction of the incident light guided by the outer segment is given by $P\left(\theta_{0}\right)=P_{0} \exp \left(-\beta \theta_{n}^{2}\right)$. For the $H E_{1, t}$ model the assumption is that the $H E_{1,1}$ mode is guided to the outer segment without loss to other guided modes or to the radiation field when $v_{B} \geqslant 1$.

The calculation of the light absorbed by the outer segment from the unguided field has been very approximate. In this respect we have chosen to avoid involved computations with specific assumptions which may not quite be justified.

The geometrical optics model is an improvement of the model by Walraven and Bouman (1960), for it uses parameters with a more evident physical interpretability. The $H E_{1,1}$ model shows some analogies with the model given by Snyder and Pask (1973). However, in their ideal average cone model the $H E_{2.1}, T E_{0.1}$ and $T M_{0.1}$ modes may be guided along the outer segment when $i<652 \mathrm{~nm}$.

Their model does not include the absorption of the radiation field by the photopigment. When certain wavelengths are considered in their ideal avcrage cone model the curve of the logarithm of the sensitivity $\eta$ as a function of the eccentricity $d$ is too flat at small values of $d$. This may be seen in Fig. 5 of their paper for $\lambda=500 \mathrm{~nm}$. Also, it may be remarked that Snyder and Pask did not explain the colour change.

There is also an analogy between the $H E_{1,1}$ model and the model by Simon (1970). When the transmission function for the wide end of the inner segment in his model is given by the transverse $E$-field of the $H E_{1.1}$ mode restrieted to a circle with area $S_{1}$, the intensity detected by the point receptor is proportional to the fraction of light $P\left(\theta_{n}\right)$ guided by the outer segment in our $H E_{1.1}$ model.

\section{CONCLUSIONS}

The model given here for the absorption of light by a cone as a function of the angle of incidence includes guided and radiated light. A consequence of the inclu. sion of the absorption from the radiated light is the nonzero limiting value of the sensitivits $\eta$ at large angles of incidence. This implies that fiting log $\|$ as a function of the angle of incidence is only possible with a parabola at relatively small angles of incidence. A Gaussian may tit the curve over a larger range /Safir and Hyams. 1969). The model was specified in two ways the geometrical opties model and the $H E$ model. The results of the measurements of Stiles $(1937)$ for the Stiles-Crawford intensity effect and for the colour change were reasonably well fitted with both models using a maximum O.D. of the visual pigment of $\left.0.565[|x|)_{\mathrm{mut}}=1.3\right]$ for the geometrical optics model and a maximum O.D. of $\left.0.738[|x|)_{\max }=1.7\right]$ for the $H E_{1,1}$ model.

The intersubject variability in the Stiles Craw ford colour effect does not necessarily imply a large intersubject difference in the optical densities of the visual pigments. In our model the colour effect is very sensitive to differences in the parameters, for example the dircctionatities $\beta$. for the cone systems

Acknowledyement - We are indebted to the Netherlands Organization for the Advancement of Pure Research (Z.W.O.) for financial support.

\section{REFERENCES}

Brindley G. S. (1953) The effects on velour vision of adaptation to very bright lights. $J$. Phtsiol 122, 332 350 .

Coble J. R. and Rushton W. A. H. (1971) Stiles Crawford effect and the bleaching of cone pigments. $J$. Physiol 217 . $231 \cdot 242$

Enoch J. M. (1960) Response of a model retinal receptor as a function of wavelength. $J$. opt. Soc. Am. $50,315-320$.

Enoch J. M. and Fry G. A. (1958) Characteristics of a model retinal receptor studied at microwave frequencies. $J$. opt. Soc. Am. 48, 899-911.

Enoch J. M. and Stiles W. S. (1961) The colour change of monochromatic light with retinal angle of incidence. Optica Acta 8, $329-358$.

King-Smith P. E. (1973a) The optical density of arythrolabe determined by retinal densitometry using the self-sereening method. $j$. Physiol. 230, 535-549.

King-Smith P. E. (1973b) The optical density of erythrolabe determined by a new method. J. Physiol 230, 551-560.

Makous W. L. (1968) A transient Stiles-Crawford effect. lision Re's. 8.1271 1284.

Mellerio 5.1971$)$ Light absorption and scatter in the human lens. Iision $R^{\prime}$ 's. 11, 129-141

Miller S. S. (1972) Psychophysical estimates of visual pigment densities in red-green dichromats: $J$ Phisiol. 223. 89.107.

O'Brien B. (1951) Vision and resolution in the central retina. J.opt. Soc tm, 41, 882-894.

Safir A. and Hyams L. 11969) Distribution of cone orientations as an explanation of the Stiles-Crawford effect. $J$. opt. Soc . Am. 59, 757.765.

Sidman R. L. (1957) The structure and concentration of solids in photoreceptor cells studicd by refractometry and interference microscopy. J. Biophis. Biochen. Cirol. 3, 15 39 
Simon 1. (1970) Un nouveau modele destiné à linterpretation de leffet Stiles-Crawford. Iision Res. 10, 1471-1476.

Smith V. C. and Pokorny J. (1972) Spectral sensitivity of color-blind observers and the cone photopigments. Vision Res. 12. 2059-2071.

Snyder A. W. (1969) Excitation and scattering of modes on it dielectric or optical fiber. IEEE Trans. MTT17. 1138 1144.

Snyder A. W. (1970) Coupling of modes on a tapered dielectric cylinder. IEEE Trans. MTT 18, 383-392.

Snyder A. W. (1972) Coupled-mode theory for optical fibers. J. opt. Soc. Am. 62, 12671277.

Snyder A. W. and Kyhl R. L. (1966) Surface mode propagation along an array of dielectric rods with all elements excited identically. IEEE Trans. AP 14.510-511.
Snyder A. W. and Pask C. (1973) The Stiles-Crawford effect explanation and consequences. $l$ ision $R e s .13,1115-113 ?$

Stiles W. S. (1937) The luminous efficiency of monochromatic rays entering the eye pupil at different points and a new colour effect. Proc. Rol: Soc. B. 123, 90-118.

Vos J. J. (1972) Literature review of human macular absorption in the visible and its consequences for the cone recep. tor primaries. Report No. IZF 1972-17. Institute for Perception R VO-TNO. Soesterberg. the Netherlands.

Wairaven P. L. and Bouman M. A. (1960) Relation between directional sensitivity and spectral response curves in human cone vision. J. opt. Soc. Am. 50. 780-784.

Weale R. A.(1961) Notes on the photometric significance of the human crystalline lens. Iision $R^{\prime} s .1,1 \times 3-191$

Wyszecki G. and Stiles W. S. (1967) Color Scintur. Wile?. New York.

Resume- Afin de retrouver les changements de couleur de Stiles-Crawford, on estime labsorption de lumières guidée et non guidée dans un segment externe de cône en fonction de langle d'incidence. On utilise soit le modèle $H E_{1,1}$ basé sur une analyse de guide d'ondes. soit un modèle d'optique géométrique. Les mesures de Stiles (1937) sont retrouvées avec un accord raisonnable, à la fois pour leffet d'intensité et pour le changement de couleur, en expliquant le changement de couleur par un effet decran prope.

Zusammenfassung-Um die Farbverschiebung nach dem Stiles-Crawford-Effekt zu erhalten, wurde die Absorption von wellenleitermoduliertem und unmoduliertem Licht im äusseren Zapfensegment als Funktion des Einfallwinkels abgeschätzt. Für ein Modell ergaben sich zwei Möglichkeiten: Das $H E_{1,1}$-Modell. das aufgrund einer Analyse der Wellenleitermoden aufgestellt wurde und das geometrisch-optische Modell. Die Messungen von Stiles (1937) wurden mit beachtlichem Erfolg bestätigt; das gilt sowohl für die Intensität als auch für dic Farbverschiebung, wobei das Phänomen der Selbstabschirmung zur Erklärung der Farbverschiebung benutzt wurde.

Резюме-Определено поглощение направленного и ненаправленного света наружным члеником колбочки, в зависимости от угла падения, для полученкя Стайл-Крауфордовского изменения цвета. Предложены две модели: $H_{1,1}$ модель основанная на волноводном модальном анализе и оптико-геометрическая модель. Измерения Стайла (1937) хоропо соответствуют как эффекту изменения интенсивности, так и изменению цвета, если для объяснения изменения цвета используется явление самоэкранирования. 